\title{
Economie institutionnelle et gestion des ressources naturelles
} renouvelables

\author{
Mr Michel Griffon
}

\section{Résumé}

L'analyse de la nature de l'appropriation des biens (biens publics, privés, communs) appliquée aux ressources naturelles renouvelables en Afrique et la notion de coût de transaction, permettent de mieux comprendre le fonctionnement des institutions et règles de gestion traditionnelles des forêts, eaux de pêche, eaux d'irrigation, pâturages, sols agricoles, etc. L'accroissement de la densité de la population rurale et de la pression d'utilisation des écosystèmes implique des changements de techniques de production et l'adaptation des institutions. Le texte explore quelques-unes des questions posées par cette évolution et conclut sur la nécessité de décentraliser l'Etat et de prendre en compte les concepts de l'économie institutionnelle pour l'évaluation des projets d'environnement.

\begin{abstract}
Institutional economics and renewable naturel resources management

The analysis of the nature of goods' property (public, private, common pool) applied to renewable natural resources in Africa and the concept of transaction costs allow a better understanding of traditional institutions and management rules of forests, fisheries, irrigation, pastures and agricultural lands. The increasing density of the rural population and the pressure on ecosystems imply changes in production techniques and institutional adjustments. This paper explores some of the issues related to those evolutions. State decentralisation appears necessary and institutional economics concepts useful for the evaluation of environment projects.
\end{abstract}

\section{Citer ce document / Cite this document :}

Griffon Michel. Economie institutionnelle et gestion des ressources naturelles renouvelables. In: Économie rurale. N²08-209, 1992. L'agriculture et la gestion des ressources renouvelables. Session des 29 et 30 Mai 1991, organisée par Maryvonne Bodiguel (CNRS) avec la collaboration de Michel Griffon (CIRAD) et Pierre Muller (CRA-FNSP) pp. 70-74;

doi : https://doi.org/10.3406/ecoru.1992.4455

https://www.persee.fr/doc/ecoru_0013-0559_1992_num_208_1_4455

Fichier pdf généré le 08/05/2018 


\title{
ÉCONOMIE INSTITUTIONNELLE ET GESTION DES RESSOURCES NATURELLES RENOUVELABLES
}

\author{
Michel GRIFFON*
}

\begin{abstract}
Résumé :
L'analyse de la nature de l'appropriation des biens (biens publics, privés, communs) appliquée aux ressources naturelles renouvelables en Afrique et la notion de coût de transaction, permettent de mieux comprendre le fonctionnement des institutions et règles de gestion traditionnelles des forêts, eaux de pêche, eaux d'irrigation, pâturages, sols agricoles, etc. L'accroissement de la densité de la population rurale et de la pression d'utilisation des écosystèmes implique des changements de techniques de production et l'adaptation des institutions. Le texte explore quelques-unes des questions posées par cette évolution et conclut sur la nécessité de décentraliser l'Etat et de prendre en compte les concepts de l'économie institutionnelle pour l'évaluation des projets d'environnement.
\end{abstract}

\section{INSTITUTIONAL ECONOMICS AND RENEWABLE NATUREL RESOURCES MANAGEMENT}

\section{Summary :}

The analysis of the nature of goods' property (public, private, common pool) applied to renewable natural resources in Africa and the concept of transaction costs allow a better understanding of traditional institutions and management rules of forests, fisheries, irrigation, pastures and agricultural lands. The increasing density of the rural population and the pressure on ecosystems imply changes in production techniques and institutional adjustments. This paper explores some of the issues related to those evolutions. State decentralisation appears necessary and institutional economics concepts useful for the evaluation of environment projects.

La théorie des choix publics (Buchanan et Tullock) a donné naissance, dans le domaine de l'économie politique des institutions, à une école de pensée très diversifiée, notamment le "Workshop in Political Theory and Policy Analysis" animé par Vincent et Elinor Ostrom à l'université d'Indiana (USA) dont les références sur la gestion des ressources naturelles renouvelables sont d'un grand intérêt, en particulier pour analyser des situations africaines.

Par les ressources naturelles renouvelables on entend : forêt, pâturages, ressources halieutiques et cynégétiques, eaux d'irrigation et de ruissellement, génotypes, sols (biotope et biocénose) et éventuellement certains écosystèmes et paysages. Quel doit être le statut juridique de ces ressources : public ou privé ? Pour les ressources communes (définition ci-après) quelles sont les règles institutionnelles qui permettent d'assurer une bonne gestion dans les ressources, c'est-à-dire d'en assurer le renouvellement et la durabilité ?

* CIRAD, URPA, Unité de Recherche Prospective Politique Agricole.

1. Travaux de recherche en cours, J. Thomson (ARD Associates in Rural Development, Inc. Burlington. Vermont), E. Ostrom, Indiana University, M.
On pourrait aussi se poser ces questions pour toute infrastructure de production ou de service, et d'une manière plus générale pour les services collectifs (1).

\section{LA PROPRIÉTÉ DES RESSOURCES NATURELLES RENOUVELABLES}

La nature de la propriété, ou de l'appropriation, ou - d'une manière encore plus large - de la relation d'appartenance d'une ressource à une société a fait l'objet de nombreuses définitions juridiques. On peut ainsi distinguer dans le droit toute une gradation dans cette relation d'appartenance, depuis l'usage à titre précaire sous conditions, jusqu'à la jouissance pleine et entière des biens (2). Il y a donc toute une série de rapports possibles entre les sociétés et les ressources, et cela génère au sein de ces sociétés des rapports sociaux

Griffon (CIRAD, Unité de Recherche Prospective et Politique Agricole). 2. Le mot "bien" est employé ici comme chose pouvant être l'objet d'un droit representant une valeur, selon la conception proposée par E. Le Roy. 
entre les membres, en fonction du rapport de chacun avec les ressources (3).

\section{Biens publics, ressources communes ou ressources privées ?}

L'apport principal des institutionnalistes américains sur l'analyse des formes d'appartenance a été de préciser les formes de la consommation ou de l'usage, et les modes d'accès à cette consommation et à cet usage.

La consommation peut être conjointe (indivisibilité) ou "en rivalité" (4) (concurrence) selon la nature de l'objet consommé. L'accès peut être contrôlable (et contrôlé ou incontrôlé) ou ouvert, libre et incontrôlable (open access). La combinaison de deux modalités des deux caractères relatifs à la consommation donne quatre types de biens :

\begin{tabular}{|c|c|c|c|}
\cline { 3 - 4 } \multicolumn{2}{c|}{} & \multicolumn{2}{c|}{ Consommation } \\
\cline { 2 - 4 } \multicolumn{2}{c|}{} & conjointe & en concurrence \\
\hline $\begin{array}{c}\text { accès ou } \\
\text { capacité } \\
\text { d'exclusion }\end{array}$ & contrôlé & biens “à péage” (5) & biens privés \\
\cline { 2 - 4 } & ouvert & biens publics & biens en commun \\
\hline
\end{tabular}

Cette technologie est bien sûr réductrice, et beaucoup de biens ne se classent pas facilement dans ces quatre catégories même si on peut les y rattacher, et beaucoup peuvent être mixtes.

Les ressources naturelles renouvelables étant pour la plupart divisibles (6), la consommation est presque uniquement "en concourrence". Ces ressources sont soit privées, soit communes. C'est à ces types de statuts que l'on s'intéresse ici. Dès lors surgit une question : les biens à accès ouvert ou libre ne peuvent concerner que des collectivités. Quelles sont-elles ? Quelles relations entretiennent-elles avec les ressources ?

\section{Les "communs" et la dimension de la collectivité concernée par l'accès à la ressource}

Dès lors qu'un individu ou un groupe contrôle l'accès - toujours lorsqu'il s'agit d'un bien "en concurrence" c'est un bien privé. Il est individuel ou collectif : familial, lignager, associatif ou appartenant à une entreprise (selon le droit local).

Si la ressource n'est pas contrôlée, ou n'est contrôlée qu'occasionnellement, pour des raisons diverses (par exemple coût d'accès élevé en raison de l'éloignement) tous ceux qui y ont accès utilisent la ressource "en commun", que la concurrence qui en résulte fasse l'objet de règles ou non. Plus une utilisation est fréquente plus les rivalités sont importantes et plus le besoin d'établissement de règles est nécessaire. Dans les zones de pâturage, par exemple, la guerre a longtemps constitué un moyen de partage (ressources fourragères et animaux). L'accroissement de la densité de population rurale augmente donc la pression sur les ressources et le domaine ouvert tend à se transformer en domaine contrôlé par le groupe. Il y aurait là, en quelque sorte, une tendance à la privatisation par le collectif.

3. Ce que Marx appelle "rapports de production et rapports sociaux de pro duction" mais que l'on réduit ici à la seule relation d'appartenance d'un bien a un individu ou un groupe. Voir L. Althusser ; E. Balibar - Lire le Capital II, p. 45. Maspero, Paris, 1970.

4. Rivalrous consumption.

5. TOLL GOODS. L'accès contrôlé permet un péage : autoroute, cinéma, télé vision par câble,...

6. Le bien est sécable en différentes parties consommables, et sa nature n'est pas affecté directement.
A l'intérieur des groupes contrôlant les ressources (par exemple contrôlant les sols d'une vallée, ou une zone de pâturage), l'accès à celles-ci peut être ouvert. Dans ce cas, les biens peuvent être considérés comme privés par "le reste du monde", car le groupe en est collectivement détenteur, mais à l'intérieur du groupe les biens sont considérés "communs" car selon la définition ci-dessus, personne ne peut en être exclu. Ce cas est fréquent dans l'histoire agraire. Ces "communs" ont pratiquement disparu en Europe et aux Etats-Unis, ils persistent sous forme de réserves ou de domaines à statut spécifique en Amérique latine. En Afrique, en revanche, ils constituent l'essentiel du domaine foncier traditionnel.

Chaque groupe humain peut ainsi disposer d'une juridiction (7), mais il se trouve aussi soumis à des obligations dues à son inclusion dans une société plus globale (par exemple en Afrique, l'ethnie et l'Etat) qui, elle aussi est une juridiction. Il y a donc emboîtement des juridictions et articulations des différentes règles coutumières et du droit entre les différents niveaux de généralité. Ainsi, un domaine commun villageois peut être considéré comme partie d'un domaine public plus vaste dont le droit qui s'y attache est défini par l'Etat, ou par une tradition coutumière plus vaste encore.

En Afrique de l'Ouest, l'accueil de migrants sur le domaine commun se fait pour des étrangers ressortissants d'ethnies et d'Etats étrangers. En fait, il existe des conventions sociales qui définissent l'accès aux ressources et les conditions d'exclusion de certains usagers. Ces conventions résultent d'évolutions historiques et sont liées aux représentations du monde qui prévalent dans les sociétés. C'est à ce point qu'il faut s'intéresser car ces représentations fondent les règles coutumières et le droit.

\section{Les représentations collectives des biens naturels} et représentations sociales peuvent faciliter ou entraver l'adaptation des techniques

L'imaginaire collectif des sociétés comporte des représentations des ressources naturelles, ainsi que des relations que les individus et sociétés ont avec elles, et des relations sociales qui sont liées à leur usage. L'analyse anthropologique des mythes, et de l'ordre symbolique des sociétés, peut permettre d'identifier les représentations collectives des ressources naturelles qui prévalent (8).

Ces représentations peuvent en effet, par l'ensemble des règles sociales qui leur sont liées, différer fortement du modèle exposé ci-dessus qui résulte de la simple prise en compte du mode de consommation des biens et du mode d'accès à ces biens. Ainsi, par exemple le contrôle de la terre peut résulter d'une conception liée au fait qu'elle est considérée comme appartenant à un dieu, ou aux ancêtres du groupe. Il est alors important de décripter la cohérence qui existe dans la société entre les représentations existantes, les règles sociales d'usage, les institutions de gestion des ressources et les problèmes réels. En effet, il peut $\mathrm{y}$ avoir un décalage entre les problèmes réels tels qu'ils existent et les modes de gestions issus de l'histoire et de la tradition.

7. Juridiction au sens américain : étendue de territoire sur laquelle un groupe fait valoir ses régles et l'appartenance du domaine au groupe.

8. On considère qu'il existe un imaginaire social, qu'il résulte d'une interprétation du réel, et qu'il génère des mythes, des rites et d'une manière générale un ordre symbolique que l'on peut analyser à travers les phénomènes religieux, les règles sociales, la tradition, la coutume et le droit. 
Dans de nombreuses régions d'Afrique, l'accroissement rapide de densité de la population et la pression sur l'utilisation des ressources naturelles renouvelables qui en résulte peut poser des problèmes que les règles traditionnelles ne peuvent pas résoudre de manière simple. L'accueil des populations allogènes sur des terroirs de plus en plus saturés pourrait donc être remis en cause et entrấner, de facto, une appropriation collective locale exclusive qui irait à l'encontre de certaines idées religieuses et du droit imposé par l'Etat pour qui la terre est nationale. La coupe du bois de feu dans les pays sahéliens entraîne des rivalités de plus en plus fortes entre villageois et coupeurs alimentant le marché urbain, posant ainsi le problème du statut des arbres, des forêts et des mythes qui s'y attachent.

La rapidité d'évolution du monde réel nécessitera des adaptations rapides du statut des ressources et de leurs modes de gestion. Ces adaptations touchent souvent en profondeur les structures de l'imaginaire collectif et peuvent provoquer des changements d'ordre culturel et social entravant ou bien facilitant les évolutions des techniques de production et des modes de gestion.

\section{LES MODES DE GESTION DES RESSOURCES NATURELLES RENOUVELABLES}

On ne s'intéresse ici qu'aux ressources communes c'està-dire en accès ouvert et avec concurrence dans l'utilisation. Le problème de gestion principal qui se pose est le suivant : pour assurer le renouvellement des ressources, quelles règles de répartition des produits et des charges faut-il instituer dans la collectivité des usagers ? Répondre à cette question implique de définir la nature précise des charges et produits en cause, et donc de définir l'ensemble des techniques de production et reproduction de la ressource. Il faut ensuite traiter des questions relatives aux règles d'accès, aux règles de recouvrement des coûts, et aux conditions de fonctionnement de l'institution de gestion.

\section{La reproduction des ressources n'est pas toujours mâ̂trisée ou connue}

L'histoire de l'utilisation des écosystèmes naturels part de la simple cueillette et va vers leur artificialisation. Pour conserver les équilibres qui assurent la durabilité de leur fonctionnement, des apports d'entretien sont nécessaires. Au-delà du simple entretien, la nature même des systèmes se transforment sous l'action de ceux qui les exploitent en introduisant des techniques nouvelles. Certaines de celles-ci affectent en profondeur le fonctionnement des agro-écosystèmes et constituent de véritables révolutions techniques. Ces changements sont de plus en plus fréquents, au fur et à mesure que les besoins d'accroissement de la production et de la productivité sont manifestes.

L'immense diversité des agro-écosystèmes fait que les utilisateurs ne disposent pas toujours de solutions techniques adaptées, mais surtout qu'ils connaissent rarement ces techniques, n'en voient pas l'utilité ou n'ont pas la possibilité de les utiliser pour des raisons économiques.

9. Certains faites réels non expliqués par les producteurs, par exemple la perte de fertilité de certains sols, peuvent faire l'objet d'interprétation mythique ou être d'une certaine manière "refoulés" car liés dans l'imaginaire à une situa-
La connaissance du fonctionnement des écosystèmes, et la conscience du rôle qu'on y joue, est une condition nécessaire pour une gestion durable des ressources. En Afrique, les coupeurs de bois ont-ils conscience de leur rôle dans l'épuisement du stock forestier ? Les éleveurs sahéliens ont-ils une connaissance suffisante des taux de charge supportables par les parcours ? Les pêcheurs connaissent-ils le mode de reconstitution des stocks de poissons ? Les agriculteurs connaissent-ils les conséquences des techniques qu'ils utilisent ? L'expérience montre que l'accès à une connaissance technique des écosystèmes est difficile, d'abord en raison de la complexité de leur fonctionnement, mais aussi parce que la compréhension met en cause des phénomènes de prise de conscience liés à la psychologie des personnes (Paulo Freire) (9), sans oublier que cette compréhension peut être incompatible avec les représentations imaginaires existantes. Cela montre la difficulté qu'il y a à proposer des institutions et des méthodes de vulgarisation et d'éducation efficaces.

Par ailleurs, la connaissance du fonctionnement des écosystèmes lorsque la pression d'utilisation est forte, renvoie de l'imaginaire collectif à la conscience individuelle. On passe d'une conception où la ressource est commune et où personne ne sent une responsabilité dans la gestion de celle-ci, à une vision où chacun peut connaître les effets de sa propre action (par exemple, la pression d'utilisation de bois de feu rend plus évident le rôle de chacun dans la réduction du stock).

\section{Les règles d'accès doivent être liées à la connaissance des écosystèmes}

Une bonne connaissance des écosystèmes permet de définir un taux de prélèvement, les conditions de prélèvement, les apports de compensation qui sont nécessaires et le coût de renouvellement.

Quelquefois, la connaissance traditionnelle suffit. $\mathrm{Au}$ Mali, dans le village de Fatola, la pêche fait l'objet de règles précises destinées à garantir les prises futures, ceci dans la partie du fleuve située entre Kayes et Moli. L'accès à la pêche est ouvert, mais à la condition de recevoir un apprentissage de deux ans de manière à connaître le comportement des poissons (emplacements selon les époques, et l'âge), à protéger leur reproduction, et à limiter les prises.

\section{Le recouvrement des coûts suppose l'existence d'une convention sociale bien établie}

Lorsque la quantité de bien commun consommé est mesurable, le paiement peut être proportionnel à la consommation : par exemple la quantité d'eau d'un puits utilisée par un troupeau, la quantité de bois coupé, l'eau d'irrigation obtenue dans un dispositif de répartition... Lorsque cette quantité n'est pas mesurable ou que le coût pour mesurer la consommation est trop élevé, les institutions procèdent souvent par taxation en distinguant éventuellement un droit d'accès (modulé selon l'appartenance au groupe ayant un accès privilégié) et un droit dont le montant peut être proportionnel à un indicateur d'utilisation, par exemple : taille de la parcelle irriguée, taille de la famille pour des travaux d'utilité commune,... 
Les accords institutionnels ou conventions passés entre usagers pour utiliser en commun les ressources et assurer leur renouvellement peuvent être très différents selon la qualité du consensus qui existe entre les membres. Classiquement, on distingue cinq sources de dysfonctionnement des conventions qui accroissent les coûts de transaction :

- information imparfaite d'une partie des membres : l'asymétrie de l'information peut donner à certains la possibilité de profiter de cette situation alors que ceux qui ne savent pas ne peuvent en profiter ;

- "sélection adverse" : on choisit d'utiliser de manière abusive le bien commun, de préferer le maximum individuel à l'optimum collectif : ceci peut intervenir, par exemple, dans l'utilisation individuelle d'une ressource commune et n'est possible que s'il n'y a pas contrôle social ;

- manquement aux règles : on choisit alors l'infraction au risque des conséquences sociales ; elle peut se développer en absence de contrôle ;

- malhonnêteté intellectuelle : elle intervient lorsqu'un usager enfreint les règles en plaidant l'ignorance de cellesci ou la bonne foi ;

- corruption : un membre qui enfreint les règles achète le silence du contrôleur ou des témoins.

L'état des institutions (leur bon ou mauvais fonctionnement) joue là un rôle important. On sait, par exemple, que les conventions qui règlent la mise en commun de l'épargne privée sont caractérisées en Afrique par des sanctions sociales très fortes en cas de manquement. Dans le domaine de la gestion des ressources, cela peut être aussi le cas : dans le village de Fatola au Mali (voir ci-dessus), un pêcheur ayant manqué aux règles en pêchant à deux reprises dans un lieu de reproduction du poisson, à une période interdite, a été battu par les membres de la communauté et a été gravement blessé.

Mais, le poids de la sanction collective ne suffit pas à conserver une convention sociale (10). Il est souvent nécessaire de consacrer les moyens pour que l'information soit parfaite, pour contrôler l'utilisation, recouvrer les coûts et diriger les actions de renouvellement. Il y a là des coûts d'organisation ou de transaction dont la charge incombe à l'ensemble des usagers : coûts de transaction nécessaires à l'établissement et l'entretien de la convention (coordination, information, contrôle) et coûts de transaction nécessaires à la reproduction de la ressource (coûts de production).

\section{Les règles d'équité peuvent conditionner \\ le fonctionnement des institutions de gestion}

Pour différentes raisons, par exemple la non maîtrise du fonctionnement des écosystèmes, ou l'information imparfaite, ou la complexité des phénomènes en cause, il peut $\mathrm{y}$ avoir inégalité des bénéfices individuels retirés d'une convention sociale.

Par exemple, toujours au Mali, au village de Sanankoro Togola (Sikasso) les institutions villageoises définissent le plan d'aménagement du terroir de manière à répartir de manière équitable les travaux collectifs de défense et de restauration des sols (bandes et diguettes en courbes de niveau), et les travaux de contrôle d'épandage des eaux de

10. Notons qu'un usager contrevenant peut être exclu, ce qui introduit un principe social d'exclusion contraire à la nature commune du bien, et qui a un coût spécifique de contrôle. ruissellement. Ces travaux sont réalisés sur plusieurs années. Il en résulte un véritable remembrement foncier et à l'issue de la redistribution, personne ne doit avoir perdu et le gain d'ensemble doit être réparti d'une manière acceptable par la collectivité.

La question est alors de savoir si les accroissements éventuels d'inégalité qui résulteraient de situations « d'asymétrie " seraient socialement acceptés ou non. Le cas du village de pêcheurs de Fatola, cité antérieurement, montre que certaines règles d'équité peuvent être introduites : à l'issue d'une journée de pêche ouverte, les prises sont mesurées et il y a redistribution partielle au profit des familles ne disposant pas de prises suffisantes.

En théorie donc, des conventions d'équité entre les différentes parties sont optimales sous deux conditions que la théorie des jeux permet de formaliser :

- les solutions sont telles que, pour tout binôme d'usagers, il n'existe pour chacun d'eux aucune solution permettant d'améliorer la position de l'un (en termes de fonction d'utilité) sans détériorer celle de l'autre (équilibre de Pareto) ;

- dans l'ensemble des solutions, celles qui sont peu équitables créent des dysfonctionnements, accroissent les coûts de transaction, et s'éloignent des équilibres de Pareto : ces solutions sont non coopératives (11).

\section{Les ressources peuvent être mises en commun pour des raisons d'économie d'échelle}

La mise en commun de biens pour des raisons d'économie d'échelle peut intervenir lorqu'un équipement artificalisant le milieu devient nécessaire, par exemple des puits pour abreuver les animaux ou des ouvrages de contrôle des eaux de ruissellement ou d'irrigation. Dès lors que différentes techniques sont possibles et s'appliquent aux usagers individuels ou bien à différentes tailles de groupes, et qu'il y a des économies d'échelle possibles, il faut faire un choix d'ordre institutionnel. Dans les projets d'irrigation par exemple, il y a quelquefois possibilité de choisir entre accès individuel à l'eau ou équipement collectif avec règles de répartition de l'eau. Evaluer a priori les coûts n'est faisable qu'en référence aux hypothèses sur le fonctionnement de l'institution chargée de gérer l'équipement. La situation optimale devrait être celle qui assure aux usagers un bénéfice maximum en tenant compte des coûts pour la collectivité en investissement, en fonctionnement et surtout en coûts de transaction liés à l'institution (coordination, information, contrôle), sachant que la convention entre les usagers doit être viable, que la ressource doit rester commune, et que l'ensemble des règles doit être acceptable en regard des traditions propres aux sociétés.

\section{CONCLUSION ET PERSPECTIVES DE RECHERCHE}

L'apport des thèses institutionnalistes est susceptible de renouveler trois domaines de réflexion importants :

- le rôle de l'Etat dans la gestion des ressources naturelles renouvelables;

- les débats sur la mise en commun des facteurs de production ;

- l'évaluation des projets.

11. Dans la mesure où l'on pourrait définir empiriquement un indicateur d'iniquité (par exemple à partir d'un coefficient de Gini), on pourrait identifier le bornage des équilibres de Pareto socialement acceptables. 


\section{La décentralisation de l'Etat est indispensable}

Dans de nombreux pays africains, comme dans les pays fortement étatisés, la faillite financière des Etats pose en termes aigus le problème de l'appropriation des ressources. L'alternative entre étatisation et privatisation est trop simpliste. La nature des ressources (type d'accès et de consommation) et la dimension du groupe des usagers concernés peuvent induire des institutions variées : des institutions vicinales ou communales, des institutions microrégionales, régionales, nationales ou supra-nationales ; il peut y avoir, en Afrique par exemple, des forêts villageoises, des forêts correspondant à des unités géographiques plus vastes incluant les centres de consommation, des forêts d'intérêt régional ou national, voire international selon l'usage auquel elles sont destinées. Le fait de définir la dimension des institutions sur la base de la dimension du groupe des usagers et de l'utilité collective réelle (principe d'agrégation des utilités) aboutit au principe de décentralisation de l'Etat au sens où les institutions ainsi définies doivent être - par construction - correctement articulées du point de vue des responsabilités.

\section{Mettre en commun ne résulte plus d'une idéologie collectiviste}

Le cadre théorique proposé pour mettre en commun les ressources ou les infrastructures et facteurs de produc- tion permettant de les gérer, permet d'articuler un calcul économique (optimisation des marges) avec un raisonnement juridique (le type d'arrangement institutionnel ou de convention), et avec un raisonnement anthropologique (les formes de l'imaginaire collectif susceptibles d'interagir avec les faits réels).

On dépasserait ainsi le débat sur l'utilité de la mise en commun des moyens de production qui a été très présent dans les économies socialistes et qui débouchait sur des impasses dues à la prééminence des choix idéologiques (12).

\section{L'évaluation des projets en termes d'économie institutionnelle est nécessaire}

Un projet de développement est en soi une institution. Beaucoup de projets se sont d'ailleurs transformés en institutions permanentes : services de vulgarisation, entreprises de gestion des eaux, sociétés d'élevage, etc. L'analyse des coûts de transaction et l'analyse en termes de théorie des jeux de l'utilité, que procurent les règles communes pour les différents usagers, ouvrent des perspectives nouvelles d'évaluation économique pour pouvoir juger de la durabilité des conventions et institutions de gestion des ressources naturelles.

12. Ainsi, dans "Calcul économique et formes de propriété", Charles Bettelheim notait que la mise en commun des moyens de production n'était efficace que si les populations avaient conscience de la nócessité de le faire : dès lors définir un objectif de collectivisation ne pouvait aboutir qu'd convaincre les usegers du bien fondé de cette collectivisation soit par exemple d'éventuelles "avant-gardes", soit par la coercition. 